\title{
New carbon stars found in the Digitized First Byurakan Survey (Research Note)
}

\author{
K. S. Gigoyan ${ }^{1}$, D. Russeil ${ }^{2}$, A. M. Mickaelian ${ }^{1}$, A. Sarkissian ${ }^{3}$, and M. G. Avtandilyan ${ }^{1}$ \\ 1 V. A. Ambartsumian Byurakan Astrophysical Observatory and Isaac Newton Institute of Chile, Armenian Branch, \\ 0213 Aragatsotn Marz, Armenia \\ e-mail: kgigoyan@bao.sci.am \\ ${ }^{2}$ Laboratoire d'Astrophysique de Marseille, CNRS-AMU, 13388 Marseille Cedex 13, France \\ e-mail: delphine.russeil@oamp.fr \\ ${ }^{3}$ Université de Versailles Saint-Quentin, CNRS/INSU, LATMOS-IPSL, 11 Bd dAlembert, 78280 Guyancourt, France \\ e-mail: alain.sarkissian@latmos.ipsl.fr
}

Received 11 May 2012 / Accepted 13 June 2012

\section{ABSTRACT}

\begin{abstract}
Faint high-latitude carbon stars (FHLCs) are distant, luminous giants. For this reason, they are often used to study the dynamical and chemical properties of the Galactic Halo. However, a more accurate investigation of photometric and spectroscopic surveys has revealed an increasing number of such objects with luminosities of main sequence stars. We report the first results of a systematic search of FHLCs using the Digitized First Byurakan Survey (DFBS) low-resolution spectral database. The DFBS low-resolution spectral plates are analysed to find new faint early and late-type carbon star candidates. Twenty new faint high-latitude carbon star candidates have been detected in an area of $\sim 4030 \mathrm{sq}$. deg. Moderate-resolution slit spectroscopy was carried out for all candidates, confirming the carbon star nature for thirteen of them (nine are new discoveries), while the remaining objects are M-type stars. The carbon star sample consists of five N-type carbon stars, for which the estimated mass-loss rates agree well with the typical ones found for such carbon stars; five comparatively bright $\mathrm{CH}$-type carbon stars; and three are probably dwarf carbon (dC) stars. The distance determination indicates that the $\mathrm{dC}$ stars belong to the thin disk, while the other stars belong the Galactic halo. These results demonstrate that one can efficiently find new faint high-latitude carbon stars in the DFBS database.
\end{abstract}

Key words. stars: carbon - methods: observational - Galaxy: stellar content

\section{Introduction}

The First Byurakan Survey (FBS) is an objective-prism spectral survey that covers the largest area to date. It is performed with the Byurakan Observatory Schmidt telescope, which covers about 17000 sq. deg. of the northern sky and part of the southern sky at high Galactic latitudes, with a total of about $2 \times 10^{7}$ objects catalogued in the entire survey. The object selection can be made according to their colours, broad emission, or absorption lines, or the spectral energy distribution (SED) to discover, classify, and investigate them. The original aim was to search for and characterize extragalactic objects, but the large amount of spectral information contained in the plates allowed a systematic search for late-type stars (Gigoyan et al. 2001, Gigoyan \& Mickaelian 2012). Up to now all results were obtained by eye inspection of the plates performed with the aid of a microscope at the Byurakan Observatory. Now, the entire plate set has been digitized, leading to the Digitized First Byurakan Survey ${ }^{1}$ (DFBS, Mickaelian et al. 2007), providing an astrometric plate solution (the typical accuracy is $\sim 1^{\prime \prime}$ ), and developing an automatic procedure to extract the spectra contained in the plates to allow a computer-based re-analysis of the data set.

Carbon (C) stars, objects with pronounced $\mathrm{C}_{2}$ and $\mathrm{CN}$ molecular bands in their spectra (for a review of carbon stars, see Wallerstein \& Knapp 1998; Lloyd Evans 2010) can be mainly

\footnotetext{
${ }^{1}$ http://byurakan.phys.uniroma1.it; http//www.aras.am/ $\mathrm{Dfbs} / \mathrm{dfbs} . \mathrm{html}$
}

divided into (i) intrinsic N-type stars and (ii) binary stars with one of the two components that has already passed through the AGB phase and pollutes its companion with C-rich material. Intrinsic $\mathrm{N}$-type carbon stars are, in general, red, luminous, variable, and on the asymptotic giant branch (AGB), for which carbon is synthesized in the core and dredged up to the stellar surface. Among the binary $\mathrm{C}$ stars are the $\mathrm{CH}$-type giants and carbon dwarfs $(\mathrm{dC})$, which are generally older than N-type objects.

The goal of this paper is to show that the DFBS is a good database to efficiently find and study early and late subclasses of the faint high-latitude carbon stars. This is illustrated here through the detection of thirteen carbon star candidates.

\section{The data}

\subsection{The Digitized First Byurakan Survey data}

The DFBS spectra have a spectral range $\sim 3400-6900 \AA$, with a sensitivity gap at $5300 \AA$ and a spectral resolution of $R=70$ in average (the dispersion is $1800 \AA / \mathrm{mm}$ near $\mathrm{H} \gamma$ and $2500 \AA / \mathrm{mm}$ near $\mathrm{H} \beta$ ). We have performed our systematic search from DFBS plates at declinations centred on $\delta=+39^{\circ},+35^{\circ},+43^{\circ}$ and $\delta=$ $+63^{\circ}$ to $+88.5^{\circ}$ (this represents 12 zones of 27 , near $\sim 4030$ sq. $\mathrm{deg}^{2}$ ). The DFBS spectral plates are analysed with the help of

\footnotetext{
${ }^{2}$ See FBS zones statistics in more detail at http://www.aras.am/ Dfbs/fbszones.htm
} 
standard image analysis softwares (FITSView and SAOImage ds9). This visualisation allows us to detect very red and faint candidate stars close to the detection limit in each DFBS plate (particularly, the range $\sim 6500-6900 \AA$ for the very late subclasses of the N-type and M-type stars, Gigoyan et al. 2010, 2009) and to perform a better selection of red objects using the possibilities of the analysis softwares compared to the eye-piece search used before (Gigoyan et al. 2001). The second and also very significant advantage is using the image analysis softwares for comparatively bright ( $m_{V} \sim 12-13 \mathrm{mag}$ ) early-type carbon stars, for which in the blue part of the low-resolution spectra the $C_{2} a b-$ sorption bands are not easy to detect due to saturation.

The investigation of the listed zones resulted in the discovery of 20 new $\mathrm{C}$ star candidates. Moderate-resolution spectroscopic observations (see Sects. 2.2 and 4) confirmed the C star nature for thirteen of them, the remaining objects are M-type stars (later than M7).

\subsection{Optical spectroscopy}

To confirm the nature of the detected candidates, moderateresolution spectroscopic observations have been performed for all of them. The observations were carried out on the $2.6 \mathrm{~m}$ telescope at the Byurakan Astrophysical Observatory (BAO, Armenia), with the SCORPIO spectrograph (Lick 3 $2063 \times 2058 \mathrm{CCD}$ with $15 \mu \mathrm{m} \times 15 \mu \mathrm{m}$ pixels). The dispersion is $1.9 \AA /$ pixel $(\delta \lambda=8 \AA$ at $6000 \AA$ ) with a wavelength coverage of 4000-7300 $\AA$.

For DFBS J064958.54+741610.1 we obtained a spectrum in the range 4300-7500 $\AA$ with the $1.93 \mathrm{~m}$ telescope equipped with the CARELEC spectrograph (300 lines/mm grating, EEV $2048 \times 1024$ CCD chip with $13.5 \mu \mathrm{m} \times 13.5 \mu \mathrm{m}$ pixels) at the Haute-Provence Observatory (OHP, France). The resulting dispersion is $1.8 \AA /$ pixel and $\delta \lambda \sim 6.7 \AA$ at $6000 \AA$.

Standard data reduction was carried out with ESO-MIDAS. All obtained spectra are presented in Figs. A.1 and A.2.

\section{Ancillary data}

\subsection{Photometry and associations}

To establish the magnitude and counterpart of the sample stars (see Table 1) we cross-correlated them with the Two Micron AllSky Point Source Catalogue (2MASS catalogue, Skrutskie et al. 2006), the USNO-B1.0 catalogue (Monet et al. 2003), the Guide

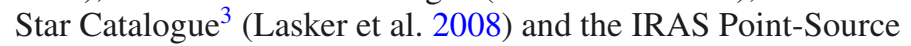
Catalogue (Beichman et al. 1989).

We also checked for known associations (search radius $2^{\prime}$ ), from the SIMBAD 4 astronomical database. Four associations were found. DFBS J020008.95+413747.4 and DFBS J230835.19+403533.9 were noted as carbon stars by Neill Reid et al. (2008) in the course of searching for ultracool dwarfs in the 2MASS catalogue. DFBS J172825.82+700829.6 was confirmed as a very distant N-type cool carbon star in the Galactic halo by Mauron et al. (2004). DFBS J064958.54+741610.1 (a late N subclasse star) was associated with FBS $0643+743$ in previous FBS lists. It was erroneously classified as an M8-M9 star (Abrahamian \& Gigoyan, 1995).

Despite the 0.3 mag uncertainty of the USNO-B1.0 magnitudes, the stars $\mathrm{n}^{\circ} 4, \mathrm{n}^{\circ} 5$, and $\mathrm{n}^{\circ} 10$ ) have $B-R>3.0$, corresponding with the typical colour of late $\mathrm{N}$-subtypes carbon stars.

\footnotetext{
3 GSC2.3, CDS catalogue I/305.

4 http://simbad.u-strasbg.fr/simbad/
}

Finally, the stars $n^{\circ} 4, n^{\circ} 5$, and $n^{\circ} 10$ exhibit IRAS counterparts among which $\operatorname{star} n^{\circ} 5$ is the reddest.

\subsection{Proper motion and variability}

We used the method previously applied for the FBS blue stellar objects by Mickaelian \& Sinamyan (2010) to calculate the proper motions of the star sample. The POSS1 and POSS2 best positions were calculated by averaging of APM/MAPS/USNOA2.0/DSS and USNO-B1.0/GSC2.32./DSS2, respectively. Knowing the epoch of observations for each object individually (from DSS1/DSS2 plates), the proper motion was calculated. In this framework, only stars $n^{\circ} 8,11$ and 13 show detectable proper motions ( $\sim 10 \mathrm{mas} / \mathrm{yr})$.

We used the Northern Sky Variability Survey ${ }^{5}$ (NSVS, Wozniak et al. 2004a) database to study the variability of the 13 new stars which have all been monitored by the NSVS. This survey provides light curves ${ }^{6}$ with a one year baseline for sources with $\mathrm{V}$ magnitudes ranging 8 to 15.5 .

Only three objects are classified as variables in the catalogue ${ }^{7}$ of Wozniak et al. (2004b): the star $n^{\circ} 3$ is classified as a semi-regular (SR) variable $(P=383$ days, $\Delta m=1.55 \mathrm{mag})$ and stars $\mathrm{n}^{\circ} 5$ and $\mathrm{n}^{\circ} 12$ as Mira-type $(\mathrm{M})$ variables $(P=356$ days, $\Delta m=3.119 \mathrm{mag}$ and $P=361$ days, $\Delta=1.772 \mathrm{mag}$ respectively). The stars $n^{\circ} 4$ and $n^{\circ} 10$ can be classified as M-type variables. All other stars have an amplitude of variability of less than $\Delta m \leq 0.2 \mathrm{mag}$.

\section{Discussion}

\subsection{Spectral types of the new objects}

Although our spectral resolution does not allow us to derive isotopic ratios, it is sufficient to detect and measure the prominent spectral features that define the carbon type by comparing our targets with those of known spectral standards presented by Barnbaum et al. (1996) and using a number of the wellknown characteristic spectral features of each group presented in Wallerstein \& Knapp (1998), Barnbaum et al. (1996), Goswami (2005), Goswami et al. (2010), Lloyd Evans (2010) and Totten \& Irwin (2000).

As a first step the general aspect of the spectrum allows us to distinguish $\mathrm{N}$-stars from $\mathrm{C}-\mathrm{R}$ and $\mathrm{CH}$-type stars. A very strong depression of light below $5000 \AA$ is a typical carbon star feature that only appears in the N-stars (Wallerstein \& Knapp 1998; Lloyd Evans 2010), and $\mathrm{H} \alpha$ tends to be filled or in emission in most N-type stars (Gray \& Corbally 2009). Inversely, early-type carbon stars show a relatively strong blue/violet continuum, but the spectra of C-R and CH-type stars are very similar (Barnbaum et al. 1996; Goswami 2005; Goswami et al. 2010).

To specify the classification we used several spectroscopic and photometric characteristics that are summarized as follows:

1. Molecular bands: strong/or weak $\mathrm{G}$ band of $\mathrm{CH}$ molecule at $4300 \AA$ with secondary P branch head at $4342 \AA$, which is the most useful indicator of the difference between $\mathrm{CH}$ and C-R stars.

2. Strong ${ }^{12} \mathrm{C}$ bands at $4737,5165,5636,6122$, and $6192 \AA$.

\footnotetext{
5 http://skydot. lanl.gov/nsvs/nsvs.php

6 The characteristics of the telescope (ROTSE-I) and the CCD used make the effective band most comparable to the Johnson $R$ band.

7 Red variables in the NSVS, http://visier.u-strasbg.fr/ viz-bin/VizieR?-source=J/AJ/128/2955
} 
Table 1. List of spectroscopically confirmed carbon stars and their photometric characteristics.

\begin{tabular}{|c|c|c|c|c|c|c|c|c|c|c|c|c|}
\hline $\mathrm{N}^{\circ}$ & DFBS Ident. & Sp. Type & $\begin{array}{c}V \\
\mathrm{mag}\end{array}$ & $\begin{array}{c}B \\
\mathrm{mag}\end{array}$ & $\begin{array}{c}R \\
\mathrm{mag}\end{array}$ & $\begin{array}{c}B-R \\
\text { mag }\end{array}$ & $\begin{array}{c}J \\
\mathrm{mag}\end{array}$ & $\begin{array}{c}H \\
\mathrm{mag}\end{array}$ & $\begin{array}{c}K_{\mathrm{s}} \\
\mathrm{mag}\end{array}$ & $\begin{array}{c}J-K_{\mathrm{s}} \\
\mathrm{mag}\end{array}$ & Var. & Notes \\
\hline 1 & J003700.13+342234.0 & $\mathrm{CH}$ & 12.29 & 14.22 & 11.27 & 2.95 & 9.484 & 8.663 & 8.438 & 1.046 & & \\
\hline 2 & J004619.15+354537.3 & $\mathrm{CH}$ & 13.61 & 15.20 & 13.00 & 2.20 & 11.795 & 11.252 & 11.115 & 0.680 & & \\
\hline 3 & J020008.95+413747.4 & $\mathrm{N}$ & 14.93 & 16.76 & 14.27 & 2.50 & 10.867 & 9.549 & 8.656 & 2.211 & Semi Reg. & (1) \\
\hline 4 & J032912.92+445330.0 & $\mathrm{N}$ & 15.06 & 19.20 & 13.50 & 5.70 & 9.157 & 7.838 & 6.875 & 2.282 & & (2) \\
\hline 5 & J064958.54+741610.1 & $\mathrm{N}$ & 15.62 & 18.59 & 12.42 & 6.17 & 9.141 & 7.526 & 6.159 & 2.982 & Mira & (3) \\
\hline 6 & $\mathrm{~J} 115153.56+412232.9$ & $\mathrm{CH}$ & 13.25 & 14.65 & 12.57 & 2.08 & 11.601 & 11.056 & 10.959 & 0.632 & & \\
\hline 7 & $\mathrm{~J} 135831.62+671042.7$ & $\mathrm{CH}$ & 13.62 & 15.22 & 12.93 & 2.29 & 11.213 & 10.514 & 10.320 & 0.893 & & \\
\hline 8 & $\mathrm{~J} 145241.15+785923.8$ & $\mathrm{CH}$ & 13.51 & 15.65 & 13.08 & 2.57 & 10.985 & 10.237 & 9.976 & 1.009 & & $\mathrm{dC}$ ? \\
\hline 9 & $\mathrm{~J} 172734.59+651110.4$ & $\mathrm{CH}$ & 12.32 & 13.72 & 11.78 & 1.94 & 9.922 & 9.195 & 8.982 & 0.940 & & \\
\hline 10 & J172825.82+700829.6 & $\mathrm{N}$ & 13.78 & 17.91 & 14.14 & 3.77 & 11.537 & 10.117 & 9.022 & 2.515 & & (4) \\
\hline 11 & J190034.62+440829.1 & $\mathrm{CH}$ & 12.10 & 13.15 & 11.06 & 2.09 & 9.951 & 9.373 & 9.146 & 0.805 & & $\mathrm{dC} ?$ \\
\hline 12 & J230835.19+403533.9 & $\mathrm{N}$ & 14.35 & 16.36 & 13.42 & 2.96 & 10.445 & 8.924 & 7.799 & 2.646 & Mira & (5) \\
\hline 13 & $\mathrm{~J} 234043.43+353104.4$ & $\mathrm{CH}$ & 14.24 & 16.11 & 13.36 & 2.75 & 11.429 & 10.685 & 10.397 & 1.032 & & $\mathrm{dC} ?$ \\
\hline
\end{tabular}

Notes. The DFBS identification (Col. 2) is coded as JHHMMSS.ss+DDMMSS.s. - (1) and (5): these stars (identified with 2MASS J02000890+4137474 and 2MASS J23083509+4035344 respectively) are rediscovered (Neill Reid et al. 2008). - (2): Identified with IRAS 03257+4443 - (3): Erroneously classified as M8-M9 subtype star in the VI list of the FBS Late-Type Stars (Abrahamian \& Gigoyan 1995). Identified with IRAS 06435+7419 - (4): Mauron et al. (2004). Identified with IRAS 17289+7010.

3. CN bands at $4215,5264,5746,6206$, and $6360 \AA$.

4. Atomic lines: BaII 4554, 4935, and $6496 \AA$.

5. Near-infrared $J H K$ photometry (see below).

As a supplementary diagnostic tool for stellar classification we used JHK photometry. Figure 1 presents a near-infrared twocolour diagram for the 13 DFBS C stars (in the SAAO photometric system obtained by transforming the 2MASS magnitudes according to Koen et al. 2007). The expected location of the C-N type (detached lines), C-CH type (solid line) and $\mathrm{dC}$ (dots) carbon stars are presented (adapted from Totten et al. 2000; Goswami et al. 2005; Goswami et al. 2010; Rossi et al. 2011). In this diagram, CH-type FHLC stars almost exclusively populate the left-hand corner.

DFBS J190034.62+440829.1, which shows complex spectra (and detectable proper motions) lies in the dwarf zone. DFBS J145241.15+785923.8 and DFBS J234043.43+353104.4 lie on the boundary between $\mathrm{CH}$ and the dwarf zone. Westerlund et al. (1995) defined dwarf carbon (dC) stars as having $J-H<0.75$, $H-K>0.25 \mathrm{mag}$. Using the transformations of Bessel \& Brett (1988), these limits become 0.76 and 0.26 , respectively, in the SAAO system, similar to that seen in Fig. 1. The JHK colours alone are not decisive evidence to guarantee membership of the $\mathrm{dC}$ star class because some fraction of the confirmed $\mathrm{dC}$ stars discovered recently (Downes et al. 2004) lies on the CH boundary region and in the boundary region between $\mathrm{CH}$-giants and $\mathrm{dC}$ stars. This is the case of PG0824+289, which is placed in the CH domain (Rossi et al. 2011) and has a composite spectrum (Heber et al. 1993), and CLS 29 as described by Totten et al. (2000). Then according to Fig. 1, DFBS J145241.15+785923.8, $\mathrm{J} 190034.62+440829.1$, and $\mathrm{J} 234043.43+353104.4$ are probably $\mathrm{dC}$ stars.

The spectral type of the thirteen candidates is listed Table 1 and the detail of the spectral classification is presented in the Appendix.

\subsection{Distance and mass loss}

\subsection{1. $\mathrm{CH}$-giants}

To calculate the distance to the CH-type stars (Table 2), we used the empirical colour-magnitude relationship obtained by

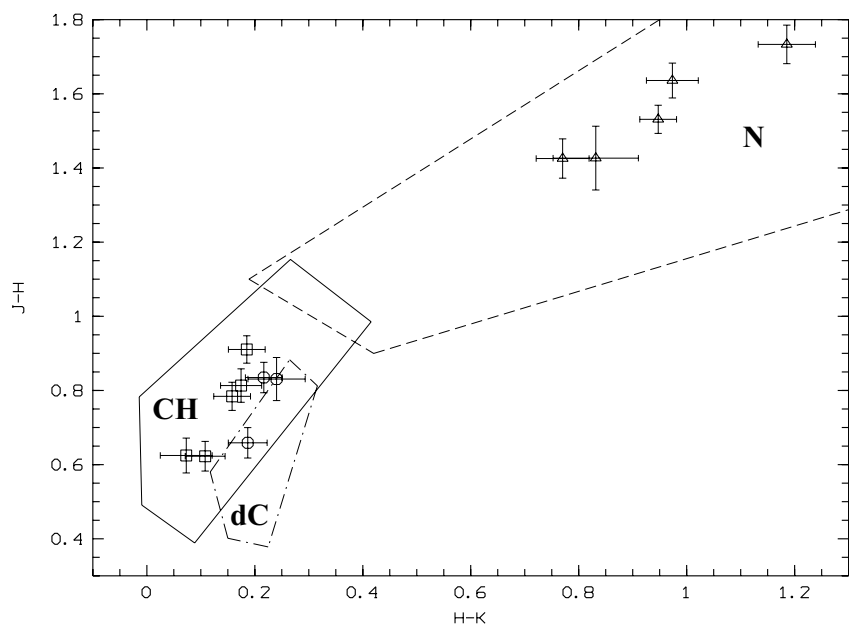

Fig. 1. Near-infrared two-colour diagram in SAAO photometric system for 13 DFBS C stars; the zones delimitating the location of the different carbon classes are those defined in the Fig. 3 by Totten et al. (2000). Open triangles are N-type stars, open squares are $\mathrm{CH}$-type stars, abd open circles are $\mathrm{dC}$ star candidates.

Totten et al. (2000) for the carbon giants located in nearby Galactic satellite systems including the dwarf satellite galaxies, LMC and SMC assuming that these satellite galaxies contain a population of carbon stars of similar nature. This relation requires $J H K$ photometry in the SAAO system. The typical uncertainty of this relation on $M_{K}$ is $\delta M_{K}=0.5 \mathrm{mag}$.

\subsection{2. $\mathrm{N}$-type stars}

To estimate the distance (Table 2) to the DFBS N-stars based on the 2MASS $K_{\mathrm{s}}$ magnitude, we used the relations given in the literature obtained recently for $\mathrm{N}$-type carbon stars.

We first determined the photometric distance using colourmagnitude relations. For each of the thirteen stars, the extinction $\mathrm{A}_{K \mathrm{~s}}$, from Schlegel ${ }^{8}$ et al. (1998), allows us to determine the $\left(J-K_{\mathrm{S}}\right)_{\mathrm{o}}$ and $(J-H)_{\mathrm{o}}$ using the relations established from Fitzpatrick (1999). Sloan et al. (2008) found for carbon stars in

${ }^{8} \mathrm{~A}_{K \mathrm{~s}}$ is taken from the NED, http: //nedwww . ipac. caltech.edu/ 
Table 2. Heliocentric distance (kpc), mass loss rates (in $10^{-7} M_{\odot} \mathrm{yr}^{-1}$ ) of N-type stars, and galactocentric $Z Y X$ coordinates (in $\mathrm{kpc}$ ).

\begin{tabular}{llccc}
\hline \hline $\mathrm{N}^{\circ}$ & DFBS Ident. & $\mathrm{D}$ & $\dot{M}$ & $Z, Y, X$ \\
\hline 1 & $\mathrm{~J} 003700.13+342234.0$ & $8.7 \pm 2.1$ & & $\mathbf{- 4 . 1}, 6.7,-12.3$ \\
2 & $\mathrm{~J} 004619.15+354537.3$ & $12.2 \pm 2.9$ & & $\mathbf{- 5 . 6 , 9 . 2 , - 1 4 . 2}$ \\
3 & $\mathrm{~J} 020008.95+413747.4$ & $19.9 \pm 2.1$ & 1.0 & $\mathbf{- 6 . 6 ,} 12.9,-22.1$ \\
4 & $\mathrm{~J} 032912.92+445330.0$ & $8.3 \pm 1.2$ & 1.3 & $\mathbf{- 1 . 4 ,} 4.1,-15.6$ \\
5 & $\mathrm{~J} 064958.54+741610.1$ & $5.9 \pm 0.6$ & 7.0 & $\mathbf{2 . 6}, 3.4,-12.6$ \\
6 & $\mathrm{~J} 115153.56+412232.9$ & $9.8 \pm 2.3$ & & $\mathbf{9 . 3}, 1.1,-11.5$ \\
7 & $\mathrm{~J} 135831.62+671042.7$ & $15.3 \pm 3.7$ & $\mathbf{1 1 . 5}, 9.3,-12.5$ \\
8 & $\mathrm{~J} 145241.15+785923.8$ & $0.054 \pm 5 \times 10^{-3}$ & $\mathbf{0 . 0 3}, 0.04,-8.5$ \\
9 & $\mathrm{~J} 172734.59+651110.4$ & $9.1 \pm 2.2$ & $\mathbf{4 . 9}, 7.6,-9.2$ \\
10 & $\mathrm{~J} 172825.82+700829.6$ & $21.8 \pm 3.1$ & 2.6 & $\mathbf{1 1 . 7}, 18.1,-11.9$ \\
11 & $\mathrm{~J} 190034.62+440829.1$ & $0.033 \pm 3 \times 10^{-3}$ & $\mathbf{0 . 0 1}, 0.03,-8.5$ \\
12 & $\mathrm{~J} 230835.19+403533.9$ & $12.9 \pm 1.2$ & 3.6 & $\mathbf{- 4 . 0}, 11.9,-11.2$ \\
13 & $\mathrm{~J} 234043.43+353104.4$ & $0.065 \pm 6 \times 10^{-3}$ & $\mathbf{- 0 . 0 3}, 0.06,-8.5$ \\
\hline
\end{tabular}

the SMC the linear colour-magnitude relation $M_{K \mathrm{~s}}=-9.18+$ $0.395\left(J-K_{\mathrm{s}}\right)\left(\delta M_{K \mathrm{~s}}=0.51 \mathrm{mag}\right)$, where $\left(J-K_{\mathrm{s}}\right)=\left(J-K_{\mathrm{s}}\right)_{\mathrm{o}}+$ 0.020 to take into account the extinction along the SMC line of sight $\left(A_{K \mathrm{~s}}=0.014\right.$ from the NED).

We extracted (thanks to the Dexter facility) a similar linear relation $\left(M_{K \mathrm{~s}}=-8.931+0.417\left(J-K_{\mathrm{s}}\right)_{\mathrm{o}}\right.$; with $\left.\delta M_{K \mathrm{~s}} 0.4 \mathrm{mag}\right)$ from Fig. 2 in Mauron (2008) from N-type carbon stars with $(J-K \mathrm{~s}) \geq 1.8$ in the LMC. Mauron (2008) also showed that carbon stars in the Sagittarius dwarf galaxy seem to follow a similar relation, but they appear to be less luminous than those of the LMC on average by $0.5 \mathrm{mag}$. Then, as in Mauron (2008), we assumed that our stars are similar to those of the Sagittarius dwarf galaxy and the adopted $\mathrm{M}_{K \mathrm{~s}}$ will be given by the relation plus 0.5 mag.

The distance can also be determined from the periodluminosity relation using the NSVS periods assuming that the 2MASS $K_{\mathrm{s}}$ magnitude is not very different from the time-averaged magnitude. For semi-regular (SR) variables we adopted Knapp et al.'s (2003) relation, while for Mira-type stars we adopted the revised Groenewegen \& Whitelock (1996) relation. We found a good agreement between the photometric and the period-luminosity distances because their mean differences is $1.4 \mathrm{kpc}$. For the three variable stars the adopted distance quoted Table 2 is the statistically averaged distance from the two methods.

In addition to the distance we evaluated the mass loss rates (in $M_{\odot} \mathrm{yr}^{-1}$ ) using the LeBertre (1997) relation. Based on modelling of the circumstellar dust shells, he found a correlation between mass loss rate and $J-K$ for $\mathrm{N}$-type carbon stars with $2 \leq J-K \leq 7$. The obtained mass loss rates (Table 2) are typical of carbon stars $\left(\sim 1.510^{-7} M_{\odot} \mathrm{yr}^{-1}\right.$, Olofsson et al. 1993). Wallerstein \& Knapp (1998) showed that on average, Mira variables have much higher mass loss rates than stars of other variable types. Although Miras with marginal mass-loss rate and semi-regular variables with pronounced winds have been observed, the approximate mass-loss rate established for stars $n^{\circ} 5$ and 12 agrees with their Mira variable type.

\subsubsection{Candidate $\mathrm{dC}$ stars}

We estimated the distance to the $\mathrm{dC}$ candidates (Table 1 ) adopting $M_{K}=+6.3$ (Harris et al. 1998), which is a mean value because dC stars with known parallaxes show $+6.1<M_{K \mathrm{~s}}<+6.5$ (Lowrance et al. 2003). This gives an $M_{V}$ of $+9.75 \pm 0.20$, $+9.29 \pm 0.19$, and $+9.89 \pm 0.20$ for stars $n^{\circ} 8, n^{\circ} 11$ and $n^{\circ} 13$, respectively. These values agree well with those of known carbon dwarfs, an exotic class of objects, which are widely accepted as binary systems.

\subsection{The galactic context}

Totten et al. (2000) argued that many of the halo carbon stars are tidal debris of merging events. Accordingly, to place the new $\mathrm{N}$-type stars in the galactic context, in particular to know if they originate from our Galaxy or from a different stellar system recently accreted by the Milky-Way, the galactocentric $X Y Z$ coordinates (see Table 2) were calculated following the convention of Newberg ${ }^{9}$ et al. (2003).

For our Galaxy, the thin disk scale height is $\sim 0.23 \mathrm{kpc}$ (Veltz et al. 2008), while the thick disk scale height is between $0.75 \mathrm{kpc}$ (de Jong et al. 2010) and $1.05 \mathrm{kpc}$ (Veltz et al. 2008). Except for the stars that have a distance $<1 \mathrm{kpc}$ ( $\operatorname{stars} \mathrm{n}^{\circ} 8,11$ and 13) and then naturally belong to the thin disk, all other stars are located beyond the thick disk $(Z>1.05 \mathrm{kpc})$.

We then looked at their position with respect to known streams and stellar overdensities (de Jong et al. 2010). The most important stream is the Sagittarius Stream (Sgr Stream), which is a sub-structure in the Galactic halo. Depending on the direction, the distance of stars in the Sgr Stream is between $16 \mathrm{kpc}$ and $50 \mathrm{kpc}$. The northern part is clearly visible at a heliocentric distance just outside $25 \mathrm{kpc}$ while its southern part is stongly detected at $l \sim 94^{\circ}$ and $178^{\circ}$ at a distance of about $20 \mathrm{kpc}$ (de Jong et al. 2010). Traced with M-type giants (Majewski et al. 2003), the Sgr Stream is a planar sub-structure approximatively given by the following equation (Newberg et al. 2003): $-0.064 X+0.970 Y+0.233 Z+0.232=0$. The Sgr Stream therefore concerns only stars beyond $16 \mathrm{kpc}$; consequently only stars $\mathrm{n}^{\circ} 3$ and 10 can potentialy belong to this stream. For these stars we then calculated a distance to the Sgr Stream plane, $d_{\mathrm{Sgr}}$, of $12.7 \mathrm{kpc}$ and $21.3 \mathrm{kpc}$, respectively. The width of the Sgr Stream being about $15 \mathrm{kpc}$ (Majewski et al. 2003), a star will belong to it if $d_{\text {Sgr }}<7 \mathrm{kpc}$. Both stars $\mathrm{n}^{\circ} 3$ and 10 have $d_{\text {Sgr }}$ much larger than $7 \mathrm{kpc}$, indicating that they do not belong to the Sgr Stream. We also checked that the stars beyond the thick disk do not belong to any other special streams/overdensities as listed by de Jong et al. (2010). We therefore conclude that they are most probably Galactic halo objects.

\section{Conclusion}

A search througth about 4030 sq. deg. area of high-latitude sky by DFBS plates yielded more than 20 carbon star candidates. Moderate-resolution CCD spectra confirm the carbon nature for thirteen of them. Nine of these thirteen stars were previously uncatalogued. The most important result is the discovery of the three $\mathrm{dC}$ star candidates, which need high-resolution investigations to quantify their possible binarity. In the same area, 33 new FBS carbon stars were previously found by eye inspection of the FBS plates. Thanks to the digitalization of the FBS survey, we have increased our sample of faint high-latitude carbon stars to $40 \%$, demonstrating that DFBS is a good database to efficiently find such stars. Downes et al. (2004) found 251 FHLC stars in more than 3000 sq. deg. of high-galactic latitudes, based on colour selection from a deep images $(15.6<r<20.8)$ survey. However, because of saturation, they were unable to access

9 The Sun is at $X=-8.5 \mathrm{kpc}, Y=0, Z=0$; the $Y$-axis is positive towards $l=+90^{\circ}$ and the $Z$ axis is positive to $b=+90^{\circ}$. 
to stars brigther than $r=14$ mag while inversly DFBS can observe them. Therefore, a systematic search for faint carbon stars using the DFBS database is in progress.

Acknowledgements. The research leading to these results has received funding from the European Community's Seventh Framework Programme (FP7/20072013) under Grant Agreement $n^{\circ}$ 228319. This research has made use of the SIMBAD database operated at CDS Strasbourg, France, and also of the Two Micron All-Sky Survey database, which is a joint project of the University of Massachusetts and the Infrared Processing and Analysis Center/California Institute of Technology.

\section{References}

Abrahamian, G. V., \& Gigoyan, K. S. 1995, Astrofizika, 38, 211

Barnbaum, C., Stone, P. S. R., \& Keenan, P. C. 1996, ApJS, 105, 419

Beichman, C., Neugebauer, G., Habing, H. J., et al. 1989, The IRAS Catalogue Of Point Sources, version 2.0, NASA-RP-1190, CDS catalogue II/125 Bessel, M. S., \& Brett, J. M. 1988, PASP, 100, 1134

de Jong, J. T. A., Yanny, B., Rix, H., et al. 2010, ApJ, 714, 663

Downes, R. A., Margon, B., Anderson, S., et al. 2004, AJ, 127, 2838

Fitzpatrick, E. 1999, PASP, 111, 63

Gigoyan, K. S., \& Mickaelian, A. M. 2012, MNRAS, 419, 3346

Gigoyan, K. S., Mauron, N., Azzopardi, M., et al. 2001, A\&A, 371, 560

Gigoyan, K. S., Russeil, D., Sarkissian, A., \& Sargsyan, L. A. 2009, Astrophysics, 52, 451

Gigoyan, K. S., Sinamyan, P. K., Engels, D., \& Mickaelian, A. M. 2010, Astrophysics, 53, 123

Goswami, A. 2005, MNRAS, 359, 531

Goswami, A., Karinkuzhi, D., \& Shantikumar, N. S. 2010, MNRAS, 402, 111

Gray, R., \& Corbally, J. 2009, Stellar spectral classification (Princeton University Press), 314
Groenewegen, M., \& Whitelock, P. 1996, MNRAS, 281, 1347

Harris, C. H., Dahn, C. C., Walker, R. L., et al. 1998, AJ, 502, 437

Heber, U., Bade, N., Jordan, S., \& Voges, W. 1993, A\&A, 267, L31

Knapp, G. P., Pourbeoix, D., Platais, I., et al. 2003, A\&A, 403, 993

Koen, C., Marang, F., Kilkenny, D., et al. 2007, MNRAS, 380, 1433

Lasker, B. M., Lattanzi, M., McLean, B. J., et al. 2008, AJ, 136, 766

LeBertre, T. 1997, A\&A, 324, 1059

Lloyd Evans, T. 2010, J. Astrophys. Astr., 31, 177

Lowrance, P., Kirkpatrick, J. D., Reid, I. N., et al. 2003, ApJ, 584, L95

Neill Reid, I., Kelle, L. C., Kirkpatrick, J. D., et al. 2008, AJ, 136, 1290

Majewski, S. R., Strutskie, M. F., Weinberg, M. D., \& Ostheimer, J. C. 2003, ApJ, 599, 1082

Mauron, N. 2008, A\&A, 482, 151

Mauron, N., Azzopardi, M., Gigoyan, K. S., \& Kendall, T. R. 2004, A\&A, 418, 77

Mickaelian, A. M., Nesci, R., Rossi, C., et al. 2007, A\&A, 464, 1177

Mickaelian, A. M., \& Sinamyan, P. K. 2010, MNRAS, 407, 681

Monet, D. G., Levine, S. E., Canzian, B., et al. 2003, AJ, 125, 984

Newberg, H. J., Yanny, B., \& Grebel, E. K. 2003, ApJ, 596, L191

Olofsson, H., Eriksson, K., Gustafsson, B., et al. 1993, ApJS, 87, 267

Rossi, C., Gigoyan, K. S., Avtandilyan, M. G., \& Sclavi, S. 2011, A\&A, 532, A69

Skrutskie, M. F., Cutri, R. M., Stiening, R., et al. 2006, AJ, 131, 1163 (Vizier Catalogue II/241)

Totten, O., Irwin, M., \& Whitelock, P. 2000, MNRAS, 314, 630

Veltz, L., Bienaymé, O., Freeman, K., et al. 2008, A\&A, 480, 753

Wallerstein, G., \& Knapp, G. R. 1998, ARA\&A, 36, 369

Westerlund, B. E., Azzopardi, M., Bretsacher, J., \& Rebeirot, E. 1995, A\&A, 303, 107

Wozniak, P. R., Vestrand, W. T., Akerlof, C. W., et al. 2004a, AJ, 127, 2436

Wozniak, S. J., Williams, P. R., Vestrand, W. T., \& Gupta, V. 2004b, AJ, 128, 2965

Schlegel, D. J., Finkeiner, D. S., \& Davis, M. 1998, ApJ, 500, 525

Sloan, G., Kraemper, K. E., Wood, P. R., et al. 2008, ApJ, 686, 1956 


\section{Appendix A: The spectral data}

In this section, we present the spectral data and the spectral classification of the star sample.

\section{A.1. Spectral description of individual star}

\section{A.1.1. The N-type stars}

Based on characteristic spectral features, five objects are classified as a N-type carbon stars. They all exhibit a similar global aspect of their spectrum with a strong depression in the blue part. Except for star $n^{\circ} 5$, all of them display $\mathrm{H} \alpha$ in emission, which is indicative of pulsations and/or mass loss.

DFBS J020008.95+413747.4 (star $\left.n^{\circ} 3\right)$ : its spectrum shows a depression of radiation below $5000 \AA$, the $\mathrm{H} \alpha$ line is in emission, and a very strong band at $6192 \AA$, due to $\mathrm{C}_{2}$ molecule, is observed.

DFBS J032912.92+445330.0 (star $\left.\mathrm{n}^{\circ} 4\right)$ : it exhibits a very low flux below $5600 \AA$ while the NaI D line is very strong.

DFBS J064958.54+741610.1 (star $\left.n^{\circ} 5\right)$ : this object shows a depression of radiation below $5500 \AA$, which is a characteristic feature of late N-subclass stars (later than N7-subtypes), and of objects with thick circumstellar envelopes. It shows hardly detectable $5636 \AA$ and $6192 \AA$ bands of $\mathrm{C}_{2}$ molecule.

DFBS J172825.82+700829.6 (star $\mathrm{n}^{\circ} 10$ ): its spectrum shows a very low flux below $5200 \AA$ and the $\mathrm{H} \alpha$ line is in emission.

DFBS J230835.19+403533.9 ( $\operatorname{star}^{\circ} 12$ ): its flux is low below $5000 \AA$ while strong features in the range 6000-6500 $\AA$, due to $\mathrm{C}_{2}$ and $\mathrm{CN}$ molecules are noted. $\mathrm{H} \alpha$ appears in emission.

\section{A.1.2. The early-type carbon stars}

Eight objects display spectra typical for early-type carbon stars, showing relatively strong blue/violet continuum. Some earlytype stars show low flux below about $4300 \AA$ that hinder an identification of the $\mathrm{CH}$ and $\mathrm{CN}$ absorption bands.

DFBS J003700.13+342234.0 ( $\left.\operatorname{star}^{\circ} 1\right)$ : a strong G-band at $4300 \AA$ with the P-branch at $4342 \AA$ is observed in the spectrum (Fig. A.1). It also shows strong features due to NaI D $(\AA)$ and $\mathrm{CN}$ bands at $5264 \AA$ А, $6206 \AA$, and $6360 \AA$.

DFBS J004619.15+354537.3 (star $\left.\mathrm{n}^{\circ} 2\right)$ : its spectrum exhibits very strong $\mathrm{C}_{2}$ and $\mathrm{G}$-band features.

DFBS J115153.56+412232.9 (star $\mathrm{n}^{\circ}$ 6): it exhibits very strong features due to the $\mathrm{C}_{2}$ molecule. The G-band and the BaII $4554 \AA$ line are intense.

DFBS J135831.62+671042.7 (star $n^{\circ} 7$ ): it exhibits strong $\mathrm{C}_{2}$ features while G-band and BaII $4554 \AA$ line are intense.

DFBS J145241.15+785923.8 (star $n^{\circ} 8$ ): for this object the G-band is hardly visible while the $6192 \AA$-band due to $\mathrm{C}_{2}$ molecule is strong.
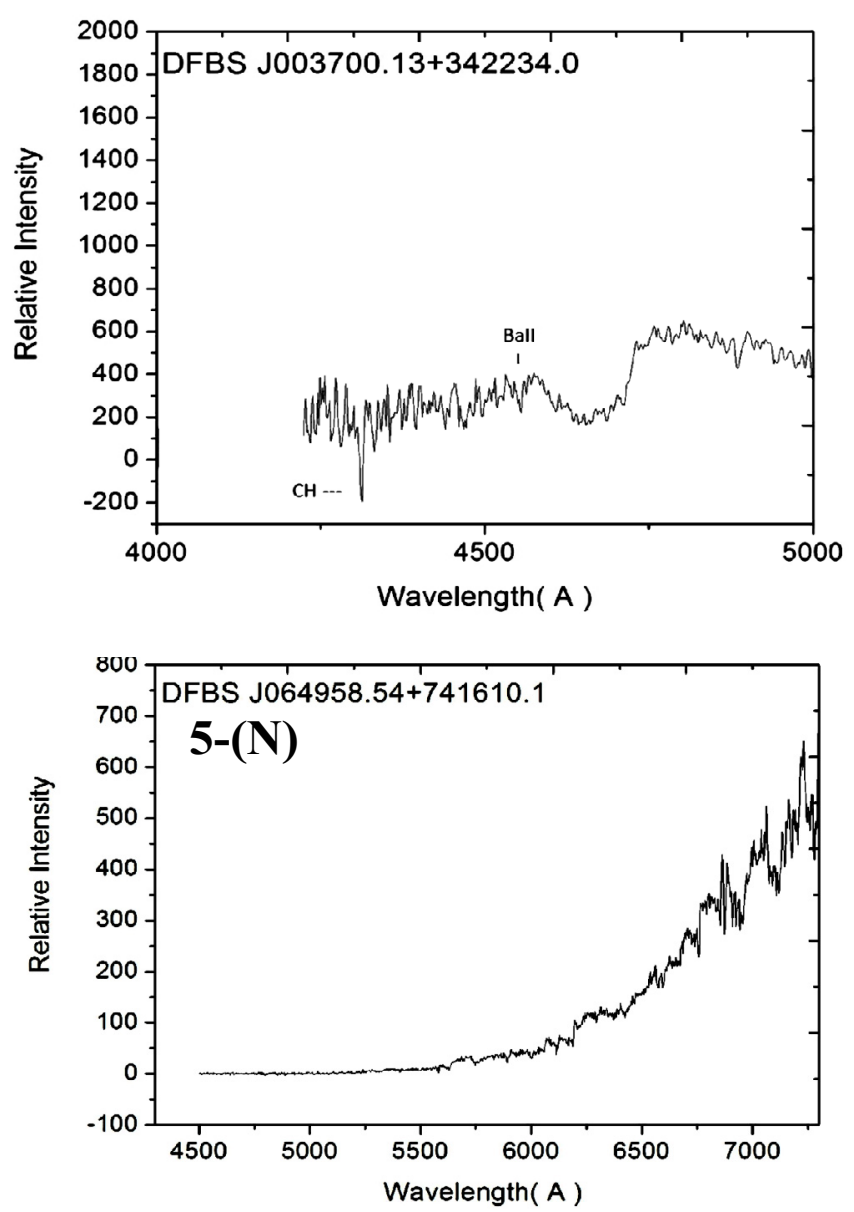

Fig. A.1. 2.6 m BAO SCORPIO spectra for DFBS J003700.13+ 342234.0 in the range 4000-5000 $\AA$ (upper panel). The $1.93 \mathrm{~m}$ OHP CARELEC spectra for DFBS J064958.54+741610.1 in the range 4300-7300 A (lower panel). Because of bad weather conditions, no standard star was observed, therefore this spectrum is not corrected for the CCD response, which explains part of the strong rising of its continuum.

DFBS J172734.59+651110.4 (star $\mathrm{n}^{\circ}$ 9): it exhibits an intense the G-band.

DFBS J190034.62+440829.1 (star $n^{\circ} 11$ ): this object shows a complex and unusual spectrum. The G-band is not visible while Na I D is very strong and Ca I $4226 \AA$ is very well expressed. This suggests that it is perhaps a binary star. The spectrum of this object looks somewhat similar to the spectrum of HE 1116-1628, known as a dC star (Goswami et al, 2010).

DFBS J234043.43+353104.4 (star $\left.n^{\circ} 13\right)$ : the identification of the $\mathrm{CN}$ and $\mathrm{C}_{2}$ bands below $4300 \AA$ is difficult due to the low flux. The $6192 \AA$-band of $\mathrm{C}_{2}$ molecule is intense. 
K. S. Gigoyan et al.: New carbon stars found in the Digitized First Byurakan Survey $(R N)$
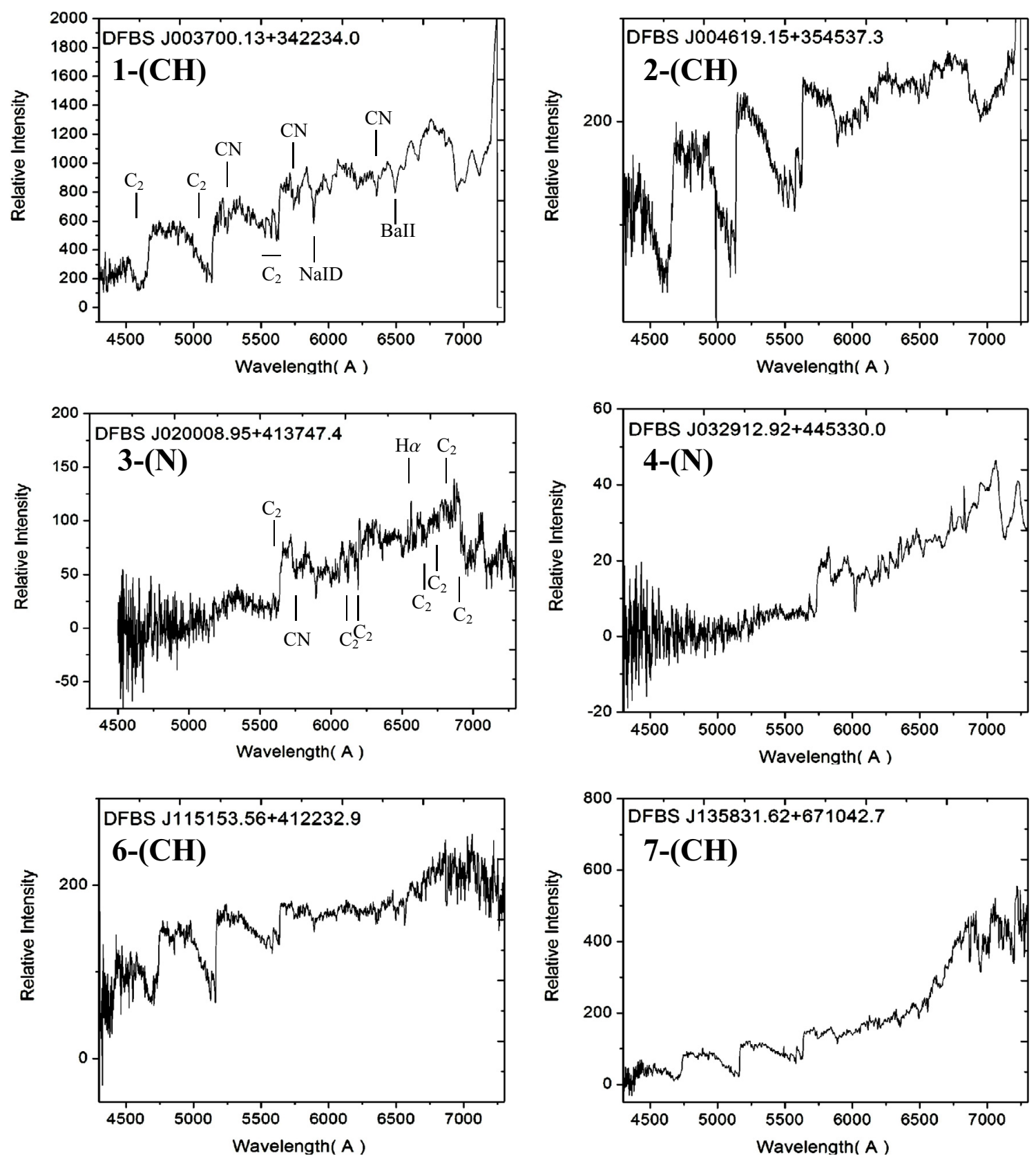

Fig. A.2. 2.6 m BAO SCORPIO (range 4300-7300 A). The main spectral features used for the classification are indicated for spectra of the stars 1 and 3 . 
A\&A 544, A95 (2012)
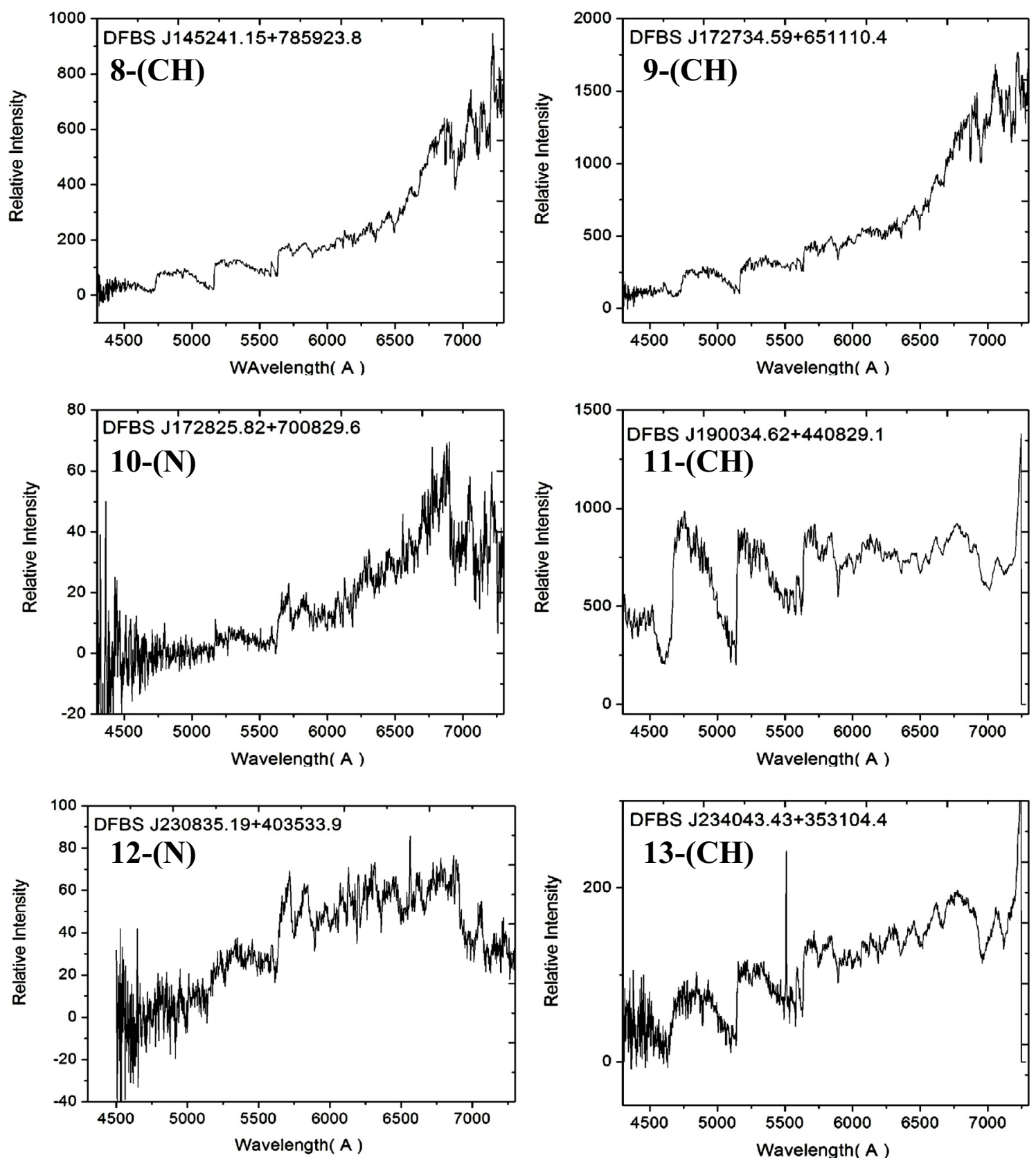

Fig. A.2. continued. 